\title{
The Interplanetary Electron Model (IEM)
}

\author{
B. Taylor, G. Vacanti, E. Maddox, C. I. Underwood
}

\begin{abstract}
A new Interplanetary electron environment model based on statistical analyses of historical datasets is presented. The model reports generates confidence limits for solar electron fluences in a similar fashion to existing Solar proton models, as well as peak event fluxes and fluences. Electrons of Jovian origin are also modeled based on simplified diffusive transport equations to provide predicted fluxes for locations within the ecliptic plane.
\end{abstract}

Index Terms - Solar Electron, Spacecraft Charging, Jovian Electrons, Space Environment

\section{INTRODUCTION}

A key part of mission planning is the estimation of the radiation environment likely to be encountered by the spacecraft. This commonly consists of the solar proton fluence, cosmic rays, and any trapped particle environments likely to be encountered. Almost invariably overlooked are electrons in interplanetary space from solar particle events and other sources. This can be attributed to the relative Total Ionising Dose (TID) and Single Event Effect (SEE) damage that may be incurred by energetic protons over electrons[1]. However, in certain scenarios the dose deposited in lightly shielded regions of a spacecraft by electrons, as well as the potential for charging can be significant, particularly in the use of cryogenics, where lower temperatures can reduce the conductivity of common space polymers. In addition, the use of highly sensitive detectors in interplanetary space (e.g: Herschal, Planck at L2) requires a good understanding of all possible sources of noise and contamination.

An extensive collection of electron flux data exists, dating back to the early 1970s. These data have been used for scientific studies of the environment, but has rarely been used to form an engineering model with predictive capabilities.

The primary source for interplanetary electron fluxes at intermediate energies $(0.2-10 \mathrm{MeV})$ is from solar flares and

Manuscript received July 22, 2011. This work was supported by the European Space Agency under ESA contract number 22915/09/NL/AF. David Rodgers acted as ESA Technical Officer on this project

Dr. Ben Taylor is with the Surrey Space Centre, University of Surrey, Guildford, UK (phone: +44 (0)1483 684711; fax: +44 (0)1483 689503; email: b.taylor@surrey.ac.uk).

Giuseppe Vacanti is with cosine Science and Computing BV, Niels Bohrweg 11, 2333 CA Leiden, The Netherlands (e-mail: gvacanti@ cosine.nl).

Dr. Erik Maddox is with cosine Science and Computing BV, Niels Bohrweg 11, 2333 CA Leiden, The Netherlands (e-mail: emaddox@cosine.nl).

Dr. Craig Underwood is with the Surrey Space Centre, University of Surrey, Guildford, UK (e-mail: c.underwood@ surrey.ac.uk)
CMEs. Secondary sources include Jovian and galactic electrons, with solar wind electrons typically falling below the $200 \mathrm{keV}$ lower limit for the model.

\section{EXISTING MODELS}

\section{A. Electron Environment Models}

Engineering interplanetary electron models have been developed by Minow et al: Solar Sail Radiation Environment (SSRE) Model and the L2 Charged Particle Environment (L2CPE) Model based on data from Ulysses and GEOTAIL respectively[2]. The Ulysses data is used in the SSRE to characterize the environment for a wide range of heliospheric latitudes.

The L2-Charged Particle Environment Model is designed to provide radiation dose estimates for spacecraft in orbit about L2. L2-CPE is an empirical engineering model for energies $<1 \mathrm{MeV}$ and is a revised version of the earlier LRAD model [3] and is based largely on data from the Geotail spacecraft.

The L2-CPE model is constructed in a similar fashion to the SSRE model, using plasma and energetic particle data from the Geotail spacecraft, rather than Ulysses. Differential flux measurements at energies between $100 \mathrm{keV}$ and $1 \mathrm{MeV}$ from the EPIC/ICS instrument on Geotail are used to constrain the high-energy end of the spectrum.

These models are based on studies of the solar wind, with more energetic particle measurements used to constrain the higher energy tails of the population. The model presented here provides a more dynamic and arguably more comprehensive study of the high energy interplanetary electron population focusing on solar particle events.

\section{B. Solar Proton Models}

Electrons associated with Solar particle events can be generated directly from a flare site, or via interactions within a CME by the same mechanism as Solar protons. As such, a review of models for much more studied Solar protons is instructive for the development of models for solar electrons.

JPL-91 is one of the standard models used for mission planning[4]. JPL-91 is based on data from the IMP series of spacecraft between 1963 and 1991 and thus comprise a large sample of the environment.

Proton events are considered as a fluence occurring over a series of days whilst the proton flux exceeds a certain threshold. The model is based solely on data collected during the solar maximum periods and assumes no SEP protons during solar minimum periods. JPL-91 is presented as a set of probability curves of exceeding a given fluence during a mission of a given duration, determined through the use of a Poisson probability distribution dependent on confidence limits and mission duration, similar to that later used by 
Xapsos et al for the ESP model[5]. Here, data from solar cycles $20-22$ from IMP $-3,-4,-5-7,-8$ and GOES $-5,-6$ and 7 were used to obtain event fluencies. The model uses a lognormal distribution as shown in (1) to predict the cumulative probability, $F_{\text {cum }}$, of exceeding a given particle fluence, $\Phi$, over a given time period. The parameters $\sigma$ and $\mu$ are dependent on the time period of $T$ active years, determined through the fitting of total annual fluence distributions. Equation 1 allows a confidence level to be calculated for any threshold energy and total fluence.

$$
F_{\text {cum }}=\frac{1}{\sigma \sqrt{2 \pi}} \int_{0}^{\Phi} \frac{1}{\Phi^{\prime}} \exp \left(-\frac{1}{2 \sigma^{2}}\left[\ln \left(\Phi^{\prime}\right)-\mu\right]^{2}\right) d \Phi^{\prime}
$$

A statistical peak flux model by Xapsos et al [6] shows that if the occurrence of solar proton events is treated as a Poisson process, a worst-case distribution for T solar active years is given by (2) and (3).

$$
\begin{aligned}
& F_{T}(\phi)=\exp \left(-N_{t o t} T[1-P(\phi)]\right) \\
& P(\phi)=\frac{\phi_{\min }^{-b}-\phi^{-b}}{\phi_{\min }^{-b}-\phi_{\max }^{-b}}
\end{aligned}
$$

Where the cumulative probability, $\mathrm{F}_{\mathrm{T}}(\phi)$ is the desired confidence level that the worst case event fluence $\phi$ will not be exceeded. $\mathrm{N}_{\text {tot }}$ is the total number of events per year having a fluence greater than or equal to $\phi_{\min }, \mathrm{b}$ is the index of the power law and $\phi_{\max }$ is the maximum event fluence. The values of $\mathrm{N}_{\text {tot }}, \mathrm{b}$ and $\phi_{\max }$ were determined through regression fits to the extensive database of proton events.

Most if not all standard solar proton models are for $1 \mathrm{AU}$ and do not include functions to propagate fluences to elsewhere in the heliosphere. A simple inverse square law of radius to vary the expected fluence in the ecliptic is commonly used [4]. Further recommendations are for fluxes to be scaled by a power law with index -3.3 for fluxes from $1 \mathrm{AU}$ to $>1 \mathrm{AU},-3.0$ from $1 \mathrm{AU}$ to $<1 \mathrm{AU}$ and fluence extrapolations to use an index of -2.5[7]. These recommendations come with the caveat to expect integer ranges around these values and are necessarily broad due to the uncertainties in propagation modes.

\section{THE INTERPLANETARY ELECTRON MODEL}

\section{A. Solar Electron Model}

As Solar electrons are generated in the same events as solar protons, the statistical formalism used in existing solar proton models is applied to electron events. A caveat to this is that there are commonly two types of solar particle event, one proton rich and the other electron rich, dependent on the exact mechanism involved[8]. In spite of this, the mechanism of generation does not have significant implications for the statistics of this model.

The work of Xapsos et al[5,6] which is applied to the ESP proton model was used as a basis to develop the formalism for the IEM solar electron model.

In order to estimate the fluence for future missions, an extensive dataset is required to form determine statistically significant parameters. An extensive review of datasets was performed to determine what data should be used in the development of IEM, considering data coverage, format, accessibility and quality. Data from instruments on the IMP-8 spacecraft was used as the primary source for this model. Data from the SOHO and ACE spacecraft were selected for use in model validation and data from ISEE-3 was used to perform early stage comparisons and checks of IMP-8 data quality.

IMP-8 was launched in 1973 into a 25-45Earth Radii orbit, well above trapped particle populations. Data collection continued until 2001, providing data over almost three solar cycles. The IMP-8 spacecraft carried a number of electron sensing instruments, the Charged Particle Measurements Experiment[9] (CPME) from Johns Hopkins University, the Goddard Medium Energy[10] (GME) experiment from GSFC and the Cosmic Ray Nuclear Composition[11] (CRNC) instrument from the University of Chicago. Data from these instruments were collected, cleaned and processed for use in the model, as described in section IV.

The IEM does not assume there are no events at solar minimum as is common for Solar proton models. Estimated rates of particle events based on data from WIND and ISEE-3 suggest an occurrence rate of $\sim 2.3$ events with enhancements above $10 \mathrm{keV}$ per month at solar minimum and $\sim 13$ events at solar maximum, providing a significantly smaller, but non zero contribution [12].

The Solar Electron model scans through a data set, and detects solar particle events by selecting periods when the particle flux rises consistently above a given threshold. This event detection requires a threshold be set for each dataset to identify the point where electrons from a solar event become the dominant source. Values for each dataset are given within the model, however to help facilitate user modifications, a histogram of the dataset is produced showing number of occurrences against flux. An example from IMP-8/GME data is given in figure 1, showing a clear background consisting of instrument noise, and electrons from secondary sources, with a high flux distribution due to solar electron events.

Data spikes are removed from the dataset by scanning for single events a given factor above the two neighbouring data points. This is defaulted to a factor 100. An upper flux threshold may also be set within the event identification algorithm to provide an upper limit for particle fluxes.

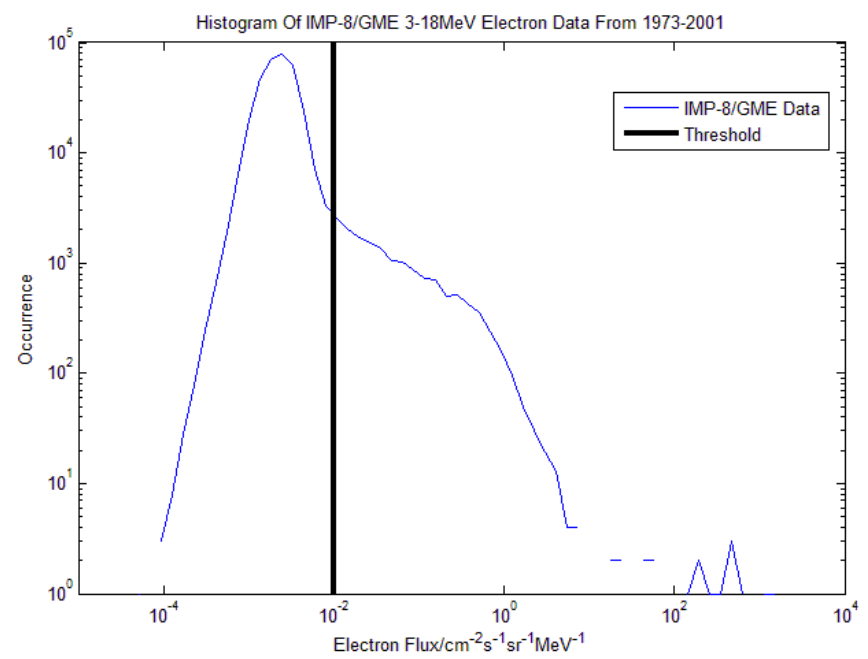

Fig. 1. Histogram profile for IMP-8/GME electron fluxes marking a threshold above which fluxes are assumed to be solely due to solar particle events 
Gaps in the data are considered differently dependent on size and whether they occur within a period determined to be part of a particle event. Small gaps occurring within a particle event, defaulted to 30 minutes, are interpolated over. Larger gaps are interpolated over if the total coverage of the event is greater than a user defined percentage, defaulted at $50 \%$.

Small gaps outside of a particle event, shorter than the duration of the shortest event are interpolated over. Larger gaps are assigned a fluence over this period equivalent to the average fluence of periods of similar duration from the rest of the dataset. This attempts to compensate for the fact that a particle event may have occurred in this time frame and been missed by the instrument.

Various parameters of these events are then calculated, ie: duration, start time, peak flux, total event fluence, etc. The total fluence at a given time resolution is then found, such as monthly or yearly. This can be done for solar maximum and solar minimum separately, to reflect the difference in event occurrence rates. The model assumes a lognormal distribution of the monthly or yearly fluence, fitting a curve to the distribution from the IMP- 8 data. This results in parameters used to determine fluence as a function of confidence level by (1).

Yearly data from IMP-8/GME and monthly data from IMP8/CPME are shown in figures 2 and 3 respectively with fitted lognormal parameters. Figure 3 uses a reorganised abscissa to better show the region of interest at higher confidence limits.

The current version of the model assumes the energetic electrons propagate diffusively and use power scaling laws based on the study of solar protons to calculate predicted fluences away from 1AU. These values are considered appropriate for use at higher particle energies; however the validity is questionable at lower energies around $200 \mathrm{keV}$. Lower energy electrons may propagate non-diffusively by focused transport due to their smaller gyro-radius and interactions with the Interplanetary Magnetic Field (IMF), leading to a more complex scaling law. A review of studies $[13,14]$ of electron transport indicates that the mode varies from event to event and an all encompassing rule would be difficult to apply.

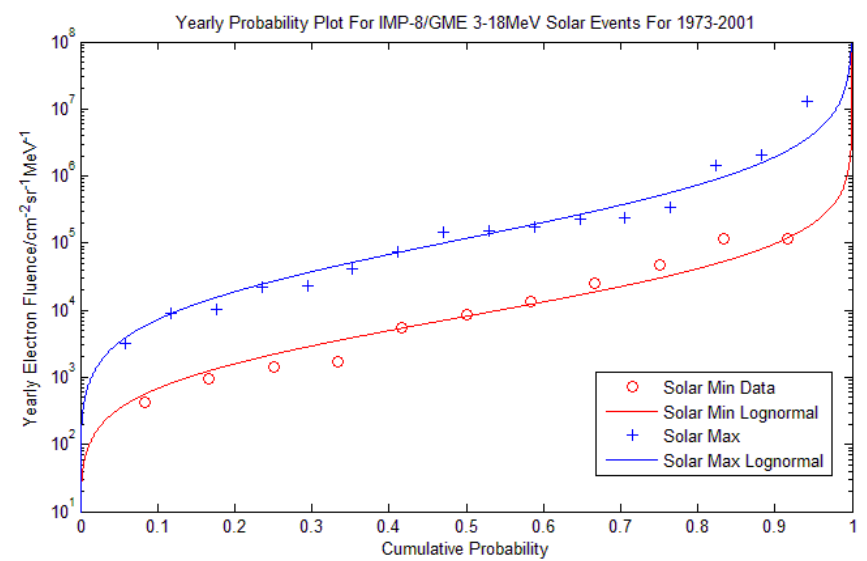

Fig 2. Confidence plot of yearly data from IMP-8/GME for 1973-2001 for solar max/min showing fitted lognormal curves

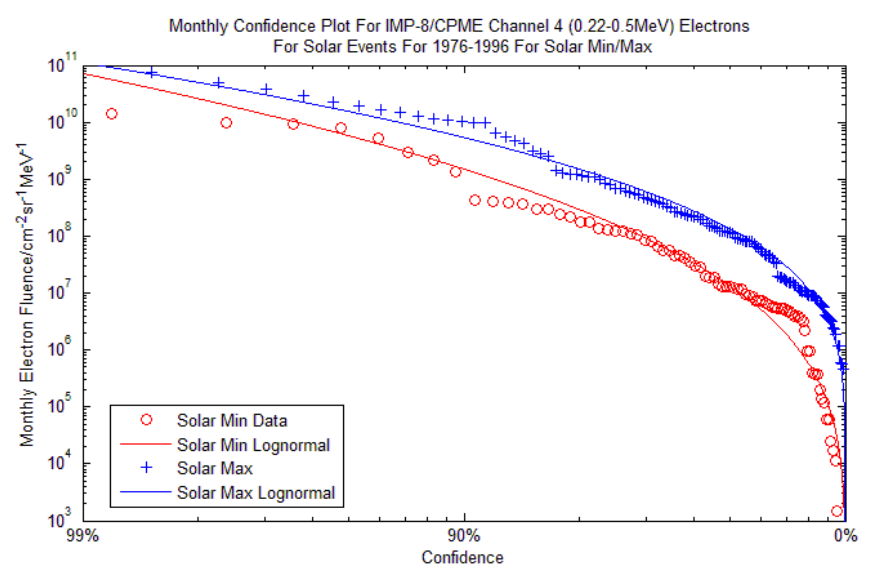

Fig. 3. Confidence plot of monthlyly data from IMP-8/GME for 1976-1996 for solar max/min showing fitted lognormal curves with a reversed logarithmic abscissa to better demonstrate higher confidence limits

Peak electron fluxes are found using a truncated power law fit to IMP-8 data as given in (2) and (3), providing confidence limits for a peak flux being exceeded for a given duration, within a given period. The peak flux data are the peak fluxes observed during individual events, with the duration over which the flux measurements should be averaged selectable by a user. This model is expected to be accurate if sufficient data is used in generating the parameters, however the model can only make predictions up to the maximum flux in this dataset due to the use of a truncated power law. This may produce a source of error at higher confidence limits due to the rare occurrence of such events. Figure 4 shows Peak flux data from IMP-8/CPME with a fitted truncated power law.

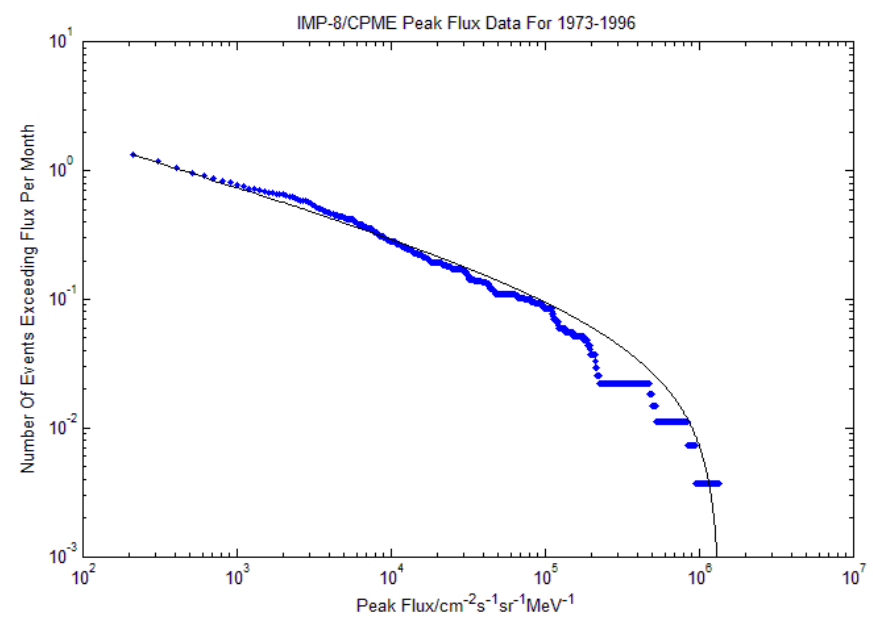

Fig. 4. Peak flux data from IMP-8/CPME for $0.22-0.5 \mathrm{MeV}$ for $1973-1996$ showing number of events exceeding a given flux per month, with truncated power law fit

The same code is also used to determine the maximum flux expected from a single event in a given period of time.

\section{B. Jovian Electron Model}

Jupiter is the primary source of energetic electrons in the Heliosphere at solar quiet time[15]. Electrons are accelerated up to $10 \mathrm{~s} \mathrm{MeV}$ within the Jovian radiation belt before leaking out of the magnetosphere and spreading throughout the 
Heliosphere. The Jovian electrons propagate diffusively along magnetic field lines and can be modeled accurately by a Fokker-Planck equation.

For the purposes of the IEM, a simplified treatment is used, allowing for an analytical solution in order to avoid computationally intensive numerical solutions[16]. This model uses a Cartesian coordinate system, mapped on to parker spirals of the Interplanetary Magnetic Field to propagate electron fluxes from Jupiter to other locations within the Heliosphere, as given in (4) with solution (5).

$$
\begin{aligned}
& \frac{\partial U}{\partial t}=\sum_{i} \frac{1}{4} \frac{\partial^{2} U}{\partial D_{i}^{2}}-F_{i} \frac{\partial U}{\partial D_{i}} \\
& D_{i}=\frac{X_{i}}{2 K_{i}^{1 / 2}}, \quad F_{i}=\frac{V_{i}}{2 K_{i}^{1 / 2}} \\
& U_{e q}(X)=\frac{A(T) \exp (2(\mathbf{D} \cdot \mathbf{F}-|D| \cdot|F|))}{8 \pi|D|\left(K_{x} K_{y} K_{z}\right)^{1 / 2}}
\end{aligned}
$$

Where $U$ is the particle density, $\mathrm{X}$ is the coordinate vector with origin at Jupiter, $\mathrm{K}$ is the diffusion tensor and $\mathrm{V}$ is the solar wind vector. Jupiter is considered as a point source generating electrons with an energy dependent function of $A(T)$.

The solar wind speed is assumed to be constant and is selectable by the user. Adiabatic energy loss is ignored in this model. At present, this model is only valid for regions close to the ecliptic plane due to a more complex structure away from this region.

Neglecting variations due to relative locations and propagation lengths between a detector and Jupiter, Jovian Electrons are modulated primarily by the $\sim 27$ solar rotation period. This is due to both blocking of electron propagation by co-rotating fast solar wind streams [17] and by enhancements of leakage from Jupiter's magnetosphere by interaction with the solar wind[18]. Further variation occurs on shorter timescales due to variations at the source. As the location of the generating coronal holes cannot be predicted ahead of time, these modulations are not incorporated into the model.

The Jovian Electron Model provides a time series envelope of electron flux below which most electron data should fall, with occasional peaks rising above this value due to occasional significant enhancements.

\section{Model Coding}

The software implementation of the model comprises two main tools, one dealing with the Jovian electrons, the other dealing with the Solar electrons. Both tools are written in $\mathrm{C}++$ and leverage common open source libraries for statistical and other data manipulations, and they are both driven by configuration files, allowing the user to vary key model parameters in response to new insights and data analysis results.

The Jovian electron tool codes the solution to the electron propagation equations described in IIIB. The intensity and spectrum of the Jovian electron source, and the diffusions coefficients affecting the electron propagation in the heliosphere can be modified by the user, although sensible defaults are given. The tool takes as input the trajectory of a spacecraft in the heliosphere, and it calculates the expected electron fluences for arbitrary energies and as a function of time.

The Solar electron tools code the formalism described in this paper. They are driven by a set of parameters $(1,2,3)$, one set of parameters encapsulating the statistical properties of the electron events in a particular energy channel of interest.

In addition to the model tools, a data analysis tool (written in Python [19]) is also provided. This can be used to access an appropriate dataset, analyze it using a range of optional parameters such as time resolution, threshold flux for solar events, etc. or use built in defaults. This tool outputs parameters to be used by the Solar electron tools to provide predictions on electron fluencies and fluxes. The data analysis tool has been designed to interface to the ESA ODI[20], so that model parameters can be updated as new electron datasets become available.

It is the intention of the ESA to make the Jovial and Solar model tools available through the SPENVIS system.

\section{IMP-8 MODEL DATA}

\section{A. Charged Particle Measurements Experiment (CPME)}

The CPME was designed and built at JHU/APL. Three solidstate detectors in an anticoincidence plastic scintillator observed electrons between 0.2 and $2.5 \mathrm{MeV}$; protons between 0.3 and $4400 \mathrm{MeV}$; alpha particles between 2.0 and $200 \mathrm{MeV}$; heavy particles with $\mathrm{Z}$ values ranging from 2 to 5 with energies greater than $8 \mathrm{MeV}$; heavy particles with $\mathrm{Z}$ values ranging between 6 and 8 with energies greater than $32 \mathrm{MeV}$; and integral protons and alphas of energies greater than $50 \mathrm{MeV} /$ nucleon, all with dynamic ranges of 1 to $1 \times 10^{6} \mathrm{~cm}^{-2} \mathrm{~s}^{-}$ ${ }^{1} \mathrm{sr}^{-1}$. Five thin-window Geiger-Mueller tubes observed electrons of energy greater than $15 \mathrm{keV}$, protons of energy greater than $250 \mathrm{keV}$, and $\mathrm{X}$-rays.

The IMP-8/CPME instrument measures electrons in the energy range $0.22-2.5 \mathrm{MeV}$ at a variable $327 / 328 \mathrm{~s}$ second resolution with a mean of 327.291s. Data were obtained from the ESAs ODI system, in .cdf format including proton data. Data are in the form of particle fluxes: FEDO (Omnidirectional Differential Electron Flux), with units $\mathrm{cm}^{-2} \mathrm{~s}^{-1} \mathrm{sr}^{-}$ ${ }^{1} \mathrm{MeV}^{-1}$. The instrument measures three distinct energy bands, combined to form a total of five channels as shown in Table 1 [10].

The CPME instrument changed functionality in August 1989 due to the failure of an anticoincidence detector used to screen for cosmic rays. This has the result of increasing the background on the electron channels, however this has no effect on the electron data itself due to significant shielding around the telescope. 
TABLE I

IMP-8/CPME CHANNEL ASSIGNMENTS

\begin{tabular}{|c|c|c|}
\hline $\begin{array}{c}\text { Channel } \\
\text { Number }\end{array}$ & $\begin{array}{c}\text { Lower Energy } \\
\text { Bound (MeV) }\end{array}$ & $\begin{array}{c}\text { Upper Energy } \\
\text { Bound (MeV) }\end{array}$ \\
\hline 1 & 0.22 & 2.5 \\
\hline 2 & 0.50 & 2.5 \\
\hline 3 & 0.80 & 2.5 \\
\hline 4 & 0.22 & 0.50 \\
\hline 5 & 0.50 & 0.80 \\
\hline
\end{tabular}

\section{B. Goddard Medium Energy (GME) experiment}

The GME instrument on IMP-6/-7/-8 measure fluxes as functions of energy and make elemental identification of protons, alpha particles and heavy ions from $<1 \mathrm{MeV} /$ nucleon to $>400 \mathrm{MeV} /$ nucleon, as well as measuring the flux of relativistic electrons in the energy range $3-18 \mathrm{MeV}$. Species identification is performed by the differential energy loss against total energy ( $\mathrm{dE} / \mathrm{dx}$ vs. E) method. The instrument consists of three particle telescopes covering the different energy ranges. Instruments on each IMP are similar, but feature a few key differences, particularly at low energies. The relevant instrument for the relativistic electrons is the Medium Energy Detector (MED) which remained mostly unchanged between spacecraft. Other detectors are used to measure protons and heavy ions, the VLET (Very Low Energy Telescope) and LED (Low Energy Detector).

The general structure of any of the IMP particle telescopes is a relatively thin front detector and one or more (thicker) following detectors, each detector capable of quantitative measurement of the energy lost by a particle in it. Collimation and anticoincidence systems are also provided to exclude/distinguish particles entering at steep angles to the detector surfaces and particles of sufficient incident energy to penetrate the detector stack. In the case of the MED, the detectors used are CsI scintillators coupled with photomultiplier tubes[9].

The IMP-8/GME instrument measures electrons in the energy range $3-18 \mathrm{MeV}$ at 30 minute resolution. Data were obtained from the NASA CDAWeb interface [21], in .cdf format including proton data.

\section{Data Considerations}

The use of data to develop a predictive model requires that they be of good quality. A number of checks were performed on the datasets used here and others considered for model inclusion or validation.

A checklist procedure has been developed in support of the IEM development. All data used in the model development and validation have been processed against this. All future datasets which may be incorporated into the model will be checked against this to ensure only good quality data are used for predictive purposes.

The data analysis checklist includes:

- Instrument Calibration - Documentation pertaining to the calibration of the instrument in question is studied to ensure published conversion factors and energy ranges are correct.
- Instrument Consistency - The full dataset is checked to ensure that there is a consistent response of the instrument to radiation. If a change in behavior is observed, the reason for this change is determined, i.e.: change in spacecraft orbit, degradation or damage to instrument, change in environment, etc. Where changes do occur, the impact on data was considered and any data not up to standard was either rejected, or included with caveats, such as the change in instrument response noted for IMP-8/CPME.

- Spatial Coverage - The spacecraft trajectory is checked and filtered to ensure that the data will not be contaminated by trapped particle populations.

- Temporal Coverage - The total time coverage of the dataset is checked to determine if inclusion in the model is worth pursuing. In addition, the number and size of gaps in the dataset is considered as to whether the dataset can provide good quality data of particle events. If there are too many data gaps to give sufficient confidence in the dataset, it is rejected.

- Cross Contamination - A significant problem for the development of a solar particle model is cross contamination of protons in electron channels. Due to the nature of these events, the electron and proton fluxes will be correlated as both species are enhanced in the course of the event. However, the ratio of electrons to protons varies significantly from event to event, and so contamination can be distinguished from correlation if a sufficiently large dataset is studied. All data are checked carefully for contamination where proton channels are available, producing a correlation plot, such as the example shown in figure 5 . This shows a strong, but variable correlation of high flux electrons and protons. At lower, a strong linear contamination can be seen. Data in this region are rejected with little impact.

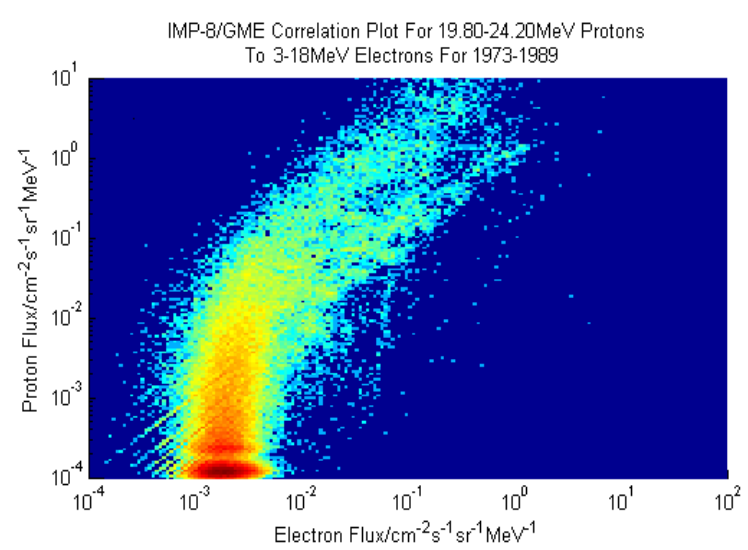

Fig. 5. Correlation plot of IMP-8/GME electrons compared to proton channel 16 containing energies of $19.8-24.2 \mathrm{MeV}$.

A fully detailed checklist is provided in the IEM user manual. 


\section{MODEL VALIDATION}

\section{A. Solar Electron Model}

During model development, data from IMP-8 were cross checked with ISEE-3/HET data[22]. The validation of the $1 \mathrm{AU}$ fluence model was performed by the comparison of predictions from the IMP-8/CPME dataset to measurements from the SOHO/COSTEP[23] and ACE/EPAM[24] instruments. Data from IMP-8/CPME channel 4 (0.22$0.5 \mathrm{MeV}$ ) from 1974-1996 were used to determine fluence thresholds for confidence limits of 50\%, 80\%, 90\% and $95 \%$ for Solar maximum and Solar minimum. SOHO/COSTEP channel is sensitive to electrons between $0.25-0.7 \mathrm{MeV}$ and so should show a reasonable match to the CPME data. Likewise, ACE/EPAM electron data are available from 0.175 $0.315 \mathrm{MeV}$. SOHO/COSTEP data are available from 19972002, with ACE/EPAM data ranging from 1997-2010. Monthly fluences for both datasets were then found via solar particle event identification procedures used for the model construction and compared to the fluence thresholds given by the IMP-8/CPME data.

The percentage of months not exceeding the fluence thresholds would be expected to be approximately the same as the confidence limit.

Figure 6 compares the fraction of events not exceeding the fluence thresholds against the confidence limits. Errors are $2 \sigma$ from Poisson statistics. These results show a good match to expectations at higher confidence limits, with $90 \%$ and $95 \%$ data falling close to the line of expectation. At lower confidence limits, a discrepancy is discernable, with the $50 \%$ confidence limit overestimating the fluence threshold. Nevertheless, all data points fall within the error bars.

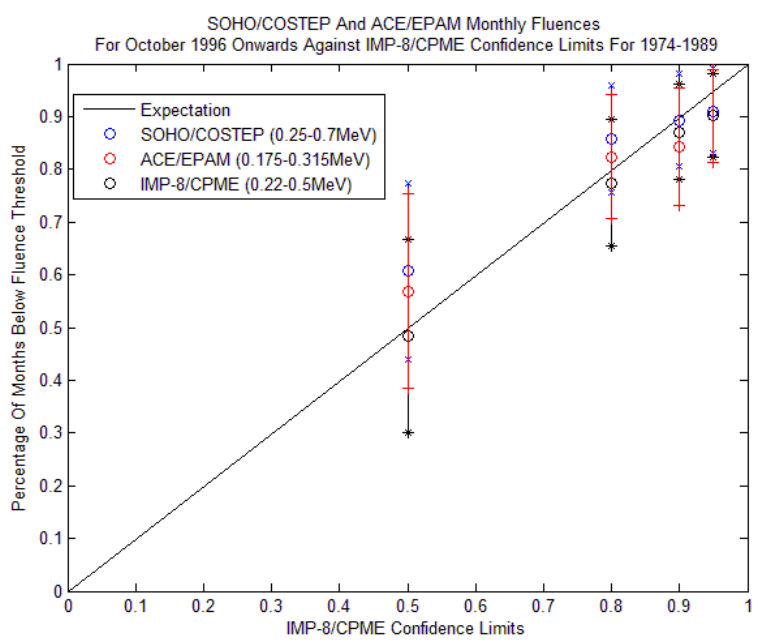

Fig. 6 Monthly SOHO/COSTEP $(0.25-0.7 \mathrm{MeV})$ and ACE/EPAM (0.175$0.315 \mathrm{MeV}$ ) fluences for the period 1996-2010 compared to expectations from IMP-8/CPME (0.22-0.5MeV)

Figure 7 shows peak flux model predictions from Figure 4 compared to monthly 5-min peak fluxes from SOHO/COSTEP and ACE/EPAM data. Also shown are predictions if the truncating maximum flux, $\phi \max$, is arbitrarily an order of magnitude greater. SOHO/COSTEP data are necessarily lower resolution compared to ACE/EPAM data due to the smaller data set, however it can seen that the shape of the confidence limit curve is tracked reasonably well above $90 \%$. The lower flux events which constitute the lower confidence limits become difficult to quantify correctly due to the short duration of these events and the possibility that they will not be identified as events within the IEM algorithms. The peak flux prediction is nevertheless greater than the fluxes at these low confidence limits and is therefore possibly conservative. At higher confidence limits above $90 \%$, the SOHO and ACE data both exceed the IMP-8/CPME derived predictions in places, primarily due to the resolution of the data. However, the ACE data include an event exceeding the largest event observed in the CPME data. A second line showing an arbitrary order of magnitude increase in the largest event seen in the CPME data shows all validation enclosed. ACE data are generally lower in the plot than SOHO data due to a longer period containing solar minimum up to 2010 where very few events were observed.

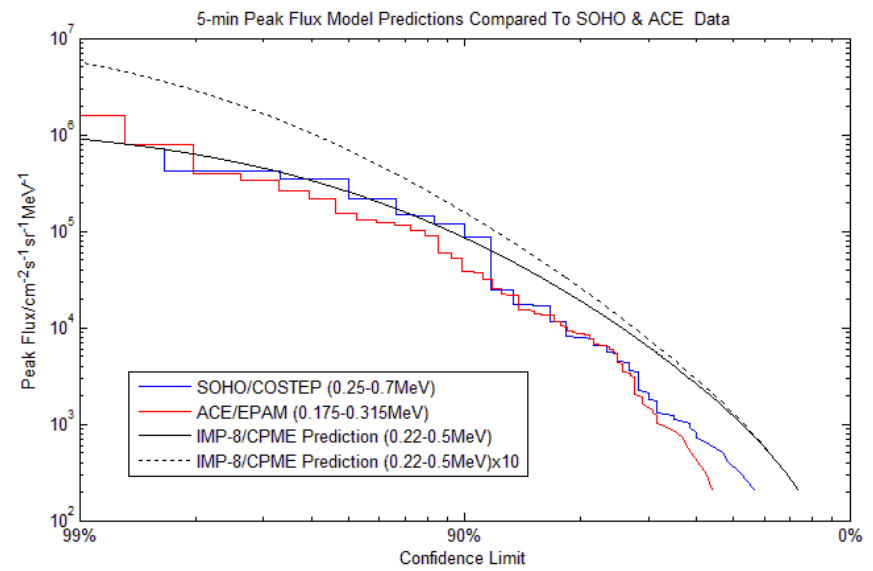

Fig. 7 Percentage of months exceeding the 5-min peak fluxes from SOHO/COSTEP and ACE/EPAM data compared to confidence levels from IMP-8/CPME data for $\phi_{\max }=$ maximum flux and $\phi_{\max }=$ maximum flux x 10

\section{B. Jovian Electron Model}

Jovian model is validated by comparing predictions against data from the IMP-8/GME instrument. IMP-8/GME detects electrons between $3-18 \mathrm{MeV}$ and so is well suited to detect electrons from Jupiter which dominate the solar quiet time population. Data from IMP-8/GME is shown in Figure 8 compared to predictions from the Jovian model at $5.84 \mathrm{MeV}$. This comparison assumes a plateau shaped energy dependent geometric factor, and using this form the Jovian spectrum power law weighted mean between $3-18 \mathrm{MeV}$ is $5.84 \mathrm{MeV}$. A background flux of $0.001 \mathrm{~cm}^{-2} \mathrm{~s}^{-1} \mathrm{sr}^{-1} \mathrm{MeV}^{-1}$ has been subtracted from the IMP-8/GME data, determined from the histogram of the flux shown in Figure 1. Solar particle events have been removed using the event finding algorithm, however the end of some of these events is still visible in the data. It can be seen that the 13-month modulation in the Jovian electron flux due to the Jupiter-Earth synodic period is fairly well matched by model predictions. The modulation can be shifted, dependent on the Solar wind speed with three values shown, $450 \mathrm{kms}^{-1}$ being approximately mean conditions. 


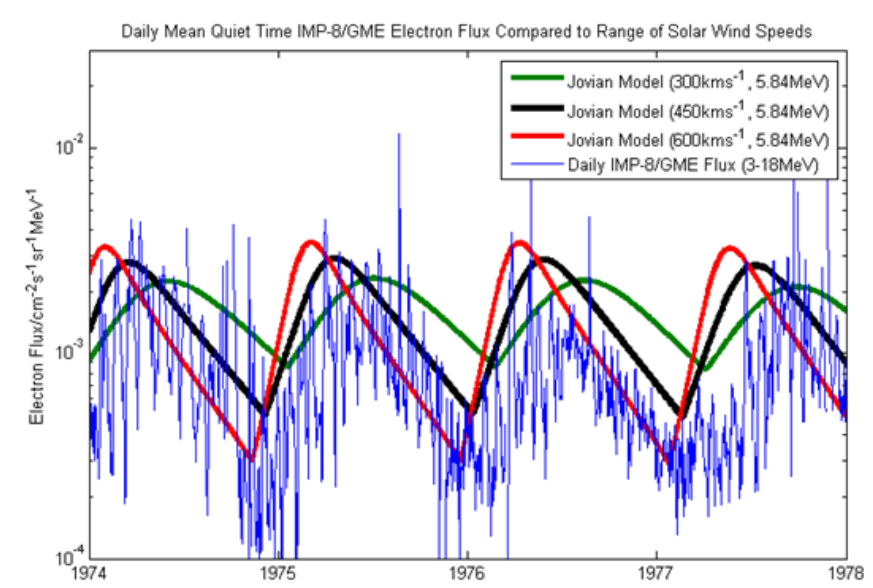

Fig. 8 Daily IMP-8/GME (3-18MeV) fluxes for the period 1974-1978 compared to predictions from the Jovian electron model for $5.84 \mathrm{MeV}$ with a variable solar wind velocity of 300,450 or $600 \mathrm{kms}^{-1}$

A comparison was also performed between Pioneer-10 data and model predictions. The CRT instrument on the Pioneer spacecraft has an electron channel at $2-6 \mathrm{MeV}$, with an average at $3.17 \mathrm{MeV}$ assuming a flat energy dependent geometric factor[25]. Figure 9 shows 6 hour resolution CRT data compared to predictions from the Jovian model based on trajectory data obtained from the National Space Science Data Center (NSSDC). In this figure, Pioneer-10/CRT data have had a background of $0.003 \mathrm{~cm}^{-2} \mathrm{~s}^{-1} \mathrm{sr}^{-1} \mathrm{MeV}^{-1}$ subtracted based on a flux histogram of the data. Data have not been cleaned for solar events and the major solar particle event of August 1972 can be seen in the data, however no other events are obvious in the data. The model again shows a good match to in-situ measurements using a solar wind speed of $450 \mathrm{kms}^{-1}$. High flux rates are observed at the end of 1973 with the close approach of the spacecraft to Jupiter and again dropped off with increasing distance.

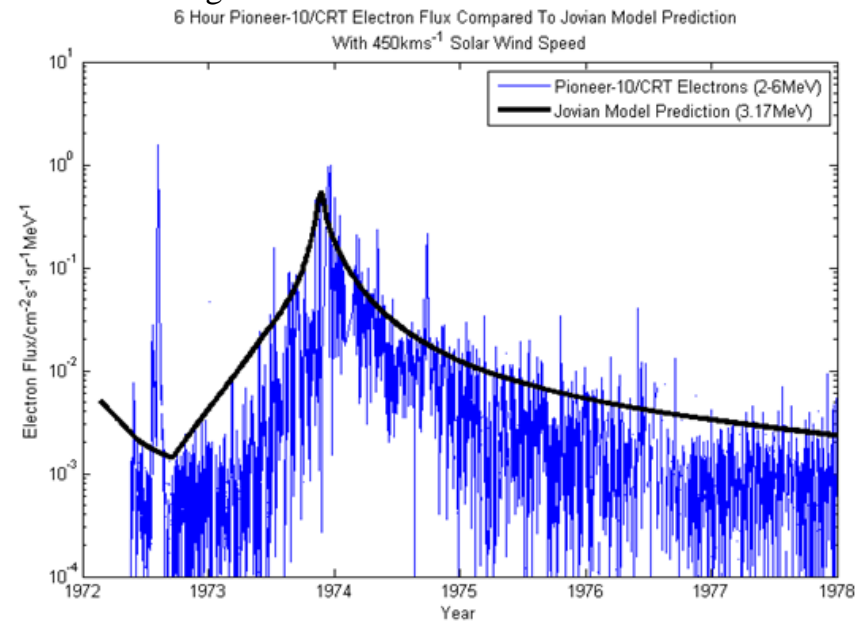

Fig. 9 Pioneer -10/CRT data from 2-6MeV for 1972-1978 compared to Jovian model predictions for $3.17 \mathrm{MeV}$.

\section{SPACECRAFT EFFECTS}

Rare peak differential electron fluxes from solar particle events near $1 \mathrm{AU}$ as measured by IMP-8/CPME are $\sim 10^{7} \mathrm{~cm}^{-}$ ${ }^{2} \mathrm{~s}^{-1} \mathrm{MeV}^{-1}$ for relatively short periods of time. This compares to trapped mean fluxes of $\sim 10^{8}$ and $\sim 10^{6} \mathrm{~cm}^{-2} \mathrm{~s}^{-1} \mathrm{MeV}^{-1}$ for typical GEO and LEO orbits respectively according to AE-8 predictions. When considering long term fluences, for a year at solar maximum CPME data provides a $90 \%$ confidence fluence of $\sim 10^{12} \mathrm{~cm}^{-2} \mathrm{MeV}^{-1}$, with a year in GEO and LEO orbits providing fluence of $\sim 10^{15} \mathrm{~cm}^{-2} \mathrm{MeV}^{-1}$ and $\sim 10^{13} \mathrm{~cm}^{-}$ ${ }^{2} \mathrm{MeV}^{-1}$. This demonstrates that even a particularly damaging year exposed to solar electrons will still be an order of magnitude less severe than for a spacecraft in a typical LEO. High energy Jovian electron fluxes at $1 \mathrm{AU}$ of $\sim 10^{-2} \mathrm{~cm}^{-2} \mathrm{~s}^{-}$ ${ }^{1} \mathrm{MeV}$ at $\sim 5.8 \mathrm{MeV}$ are orders of magnitude lower than those found at GEO altitudes, on the order of $\sim 10^{0} \mathrm{~cm}^{-2} \mathrm{~s}^{-1} \mathrm{MeV}$.

From the data presented in this paper, it can be seen that the interplanetary electron environment is fairly benign when compared to the more commonly considered sources of ionizing radiation such as trapped protons and electron and solar protons. TID from a long term fluence of solar electrons is low compared to that from trapped particle populations in Earth orbit. Electron fluxes for large particle events are however not so low that they can be disregarded from a spacecraft charging perspective however, as evidenced by peak event fluxes typical of those encountered in the trapped electron environment.

\section{SUMMARY}

An Interplanetary Electron Model has been developed to provide data on solar particle events and Jovian electrons. The tool is updatable as new data becomes available. The IEM is intended for use as an engineering tool and as such the model does not require a substantial understanding of the subject to operate. However, the model does incorporate more advanced functionality, resorting to default settings when this is not required.

At present a number of limitations exist. The solar electron model currently does not provide a particle spectrum automatically and this must be generated from a number of datasets. Coverage is provided between $0.2-10 \mathrm{MeV}$ by the three instruments on IMP-8, however more datasets should be added to improve the quality of such a function.

Propagation of electron fluxes around the solar system is limited in the model at present, due to the range of modes of propagation of electrons at these energy ranges.

Ultimately, the model limitations are due to insufficient data outside of the Earth's magnetosphere. As more data is generated by interplanetary craft, it is expected that the quality of the IEM can improve.

At present the IEM is a set of command line programs. It is intended that ultimately the model will be integrated into the online SPENVIS suite to complement existing Solar proton models, resulting in a more user friendly GUI system.

\section{ACKNOWLEDGMENTS}

The authors would like to thank ESA for providing funding for the IEM project and David Rodgers for his support as ESA Technical Officer.

The Authors would also like to acknowledge the teams that generated and provided the data use in the development of this model. These are the National Space Science Data Center, NASA Goddard Space Flight Center's CDAWeb, and ESA's 
ODI system. For the invaluable data provided from their instruments, we would like to thank: Dr. Robert E. McGuire, PI for IMP-8/GME; Dr. Robert B. Decker, PI for IMP8/CPME; Dr. Clifford Lopate, PI for IMP-8/CRNC; Dr. Tycho T. Von Rosenvinge, PI for ISEE-3/HET; Dr. Horst W. Kunow, PI for SOHO/COSTEP; Dr. Robert E. Gold, PI for ACE/EPAM and Dr. Frank B. McDonald, PI for Pioneer10/CRT.

\section{REFERENCES}

[1] Holmes-Siedle, A., Adams, L., "Handbook of Radiation Effects", Oxford Science Publications, ISBN: 0-19-856347-7, 1993;

[2] Minow, J.I., Neergard Parker, L., Altstatt, R.L., Blackwell, W.C. Jr., Diekmann, A., "Radiation and internal charging environments for thin dielectrics in interplanetary space", Proc. of 9th Spacecraft Charging Technology Conf., Tsukuba, 2005;

[3] Minow, , J.I., Neergard Parker, L., Altstatt, R., Skipworth, W., "Plasma environment and models for L2", 44th AIAA Aerospace Sciences Meeting and Exhibit, 2004;

[4] Feynman, J., Spitale, G., Wang, J. and Gabriel, S., "Interplanetary Proton Fluence model: JPL 1991" J. Geophys. Res., 98 A8, pp. 13,28113,294, 1993;

[5] Xapsos, M.A, Summers, G.P., Barth, J.L., Stassinopoulos, E.G., Burke, E.A.," "Probability Model for Cumulative Solar Proton Event Fluences", Trans. Nucl. Sci., vol. 47, no. 3, 2000;

[6] Xapsos, M.A, Summers, G.P, Burke, E.A.," Probability Model for Peak Fluxes of Solar Proton Events", Trans. Nucl. Sci., vol. 45, no. 6, 1998;

[7] Feynman J., Gabriel, S., "Interplanetary Particle Environment, Proceedings of a conference", 1989;

[8] Cliver E.W., Ling, A.G., "Electrons and Protons in Solar Energetic Particle Events", Astrophys. J. 658, 1349-1356, 2006;

[9] Armstrong, T.P., et al., "Magnetosheath Bursts of Predominantly Medium Nuclei Observed with IMP-8 on February 16, 1974", J. Geophys. Res., v. 83, p. 5198, 1978.

[10] McGuire, R., http://spdf.gsfc.nasa.gov/imp8 GME/GME instrument.html, last updated 1996, accessed on $1^{\text {nd }}$ October 2010;

[11] Novikova, E.I., Dietrich, W.F., Tylka, A.J., Collins, J., Phlips, B.F., "Monte Carlo calibration of the response of the University of Chicago's Cosmic Ray Nuclei Experiment (CRNE) on IMP-8 to electrons above 0.5MeV", Adv. In Space Res., vol. 46, pp31-43, 2010;

[12] Nieminen, P., "On the energy spectra and occurrence rate of solar electron events", Proc. of Space Radiation workshop, DERA, Nov. 1999 ;

[13] Wibberenz, G., Cane, H.V., "Multi-spacecraft observations of solar flare particles in the inner heliosphere", Astrophys. J., vol. 650, p. 1199, 2006;

[14] Beeck, J.; Mason, G. M.; Hamilton, D. C.; Wibberenz, G.; Kunow, H.; Hovestadt, D.; Klecker, B. "A multi-spacecraft study of the injection and transport of solar energetic particles", Astrophys. J., Part 1, vol. 322, pp. 1052-1072, 1987;

[15] Chenette, D. L.; Conlon, T. F.; Pyle, K. R.; Simpson, J. A., "Observations of Jovian Electrons at 1AU throughout the 13 Month Jovian Synodic Year", Astrophys. J., Part 2 - Letters to the Editor, vol. 215, 1977, L95-L99.;

[16] Conlon, T. F. ,'The interplanetary modulation and transport of Jovian electrons", J. Geophys. Res., vol. 83, p. 541-552, 1978;

[17] Conlon, T. F.; Simpson, J. A., "Modulation of Jovian Electron Intensity in interplanetary space by co-rotating interaction regions", Astrophys. J. 211, pt. 2, p. L45-L49, 1977;

[18] Chenette, D. L.; "The propagation of Jovian electrons to Earth", J. Geophys. Res., 85(A5), 2243-2256, 1980;

[19] http://www.python.org accessed on 31/01/2011;

[20] http://www.lund.irf.se/odi/ accessed on 31/01/2011;

[21] http://cdaweb.gsfc.nasa.gov/ accessed on 20/07/2011;

[22] Von Rosenvinge, T.T., McDonald, F.B., Trainor, J.H., Van Hollebeke, M.A.I., Fisk, L.A., "The Medium Energy Cosmic Ray Experiment for ISEE-C", IEEE Trans. Geosci. Elec., vol GE-16, no. 3, pp 208-212, July 1978 ;

[23] Muller-Mellin, R., et al, "COSTEP- Comprehensive Suprathermal and Energetic Particle Analyser”, Solar Physics, vol 162, pp 483-504, 1995;
[24] Gold, R.E., Krimigis, S.M., Hawkins, S.E., Haggerty, D.K., Lohr, D.A., Fiore, E., Armstrong, T.P., Holland, G., Lanzerotti, L.J., "Electron, Proton and Alpha monitor on the compostion explorer spacecraft", Space Sci. Rev., vol. 86, pp. 541-562, 1998;

[25] Stilwell, D. E.; Joyce, R. M.; Teegarden, B. J.; Trainor, J. H.; Streeter, G.; Bernstein, J., "The Pioneer 10/11 and Helios A/B cosmic ray instruments", IEEE Trans. Nucl. Sci., vol. NS-22, pp. 570-574, 1975; 